The well-known Prisoner's Dilemma is the most common game theory paradigm. It proves that 2 individuals who act in their own self-interest do not produce the optimal outcome. As countries try to secure vaccine availability for their citizens, they may fail to slow the spread of the virus elsewhere, which harms human society overall. For example, if Germany concentrates on making the vaccine available only for all German citizens while the outbreak grows in Belgium, Switzerland, and other neighboring countries, a shortage of ICU beds could result in these countries. However, such a shortage led to critical care patients being transferred to Germany, where the ICU capacity was sufficient.

The Tragedy of the Commons refers to bad outcomes for the whole system, when individual self-interests conflict with the common good. It is based on a 200 -year-old concept that originally described a group of shepherds who let their sheep graze on a shared field, resulting in the grass getting eaten down to the roots and, thus, all sheep dying. As more and more "wealthy" countries order large numbers of vaccines for their citizens, concerns arise that, if the manufacturing capacity limit is reached, low-income countries may have to wait $2-3$ years to get vaccines. The COVAX initiative aims to prevent such vaccine hoarding by guaranteeing vaccines to $20 \%$ of each country's most vulnerable population. Such an approach gives shepherds (in this case the pharmaindustry) time to plant new grass (in this case more vaccines) for the sheep (in this case the population). Moreover, approval of $>1$ effective vaccine means more grass for a given number of individuals.

The Free Rider Dilemma refers to an individual or a group who uses a resource or receives a benefit without having any contribution to it. In the COVID-19 era, free riders can be considered all persons that benefit from herd immunity without exposing themselves to the vaccine. At the country level, free riders can be considered countries that have not participated in the negotiations for the vaccine distribution (eg, countries that will receive vaccines just because they are EU members) or developing countries that do not participate in the COVAX facility but take advantage of its decisions.

In the Volunteer's Dilemma, he who goes first loses, but if no one tries all lose. For example, University of Oxford/AstraZeneca (Oxford/AZ) partnership was the first to conclude a vaccine agreement with the European Commission. ${ }^{3}$ Oxford/AZ promised vaccine at uniform price worldwide $(\$ 2.90)$ and was granted protection from future product liability claims. The Pfizer/ BioNTech vaccine was approved for use in the United Kingdom, and although it is priced at $\$ 20.00$, the UK government also granted the manufacturer protection from being sued. ${ }^{4}$ This example may also indicate reciprocity - a situation in which, after adopting a cooperative strategy on the first move, the player proceeds by copying the moves of the other players.

Finally, the Stag Hunt Dilemma describes a conflict between safety and cooperation. According to the scenario, a group of hunters go hunting. If all hunters work together and kill the stag, it will provide shared meat for all. If they work at their immediate self-interest, they may succeed killing a hare (which is worth less than a stag), providing adequate meat only for the individual. The problem is that there is no guarantee for a stag, so the hunter's dilemma when he sees a hare is whether to kill it or to sacrifice his immediate self-interest for the risk of not eating anything. In the COVID-19 era, the stag is a highly effective vaccine, which, at the beginning, could not be guaranteed. Oxford/AZ reported an overall vaccine efficacy of $70 \%$. To increase this efficacy, Oxford/AZ decided to work with the makers of Russia's Sputnik-V vaccine to test a combined shot, based on 1 of 2 vectors of Sputnik-V. This could produce greater and longer-lasting immunity.

In conclusion, human cooperation is the only driving force that can minimize such dilemmas and provide a fair, uniform, and equitable COVID-19 vaccine allocation.

\section{Acknowledgments.}

Financial support. No financial support was provided relevant to this article.

Conflicts of interest. All authors report no conflicts of interest relevant to this article.

\section{References}

1. Greer SL. National, European, and global solidarity: COVID-19. Eurohealth 2020;26:104-108.

2. McFadden DW, Tsai M, Kadry B, Souba WW. Game theory: applications for surgeons and the operating room environment. Surgery 2012;152:915-922.

3. Ng WH, Liu X, Mahalingam S. Development of vaccines for SARS-CoV-2. F1000Res 2020. doi: 10.12688/f1000research.25998.1.

4. Hafner M, Yerushalmi E, Fays C, Dufresne E, Van Stolk C. COVID-19 and the Cost of Vaccine Nationalism. Santa Monica, CA: RAND Corporation; 2020.

\title{
Why comparing coronavirus disease 2019 (COVID-19) and seasonal influenza fatality rates is like comparing apples to pears
}

\author{
Marios Papadakis MD, MBA, PhD (10 \\ University Witten-Herdecke, Witten, Germany
}

To the Editor-The coronavirus disease 2019 (COVID-19) pandemic is a once-in-a-lifetime event for humanity. By the end of March 2021, 130 million cases had been confirmed worldwide and $>2.8$ million people have died, with a case fatality rate (CFR)

Author for correspondence: Marios Papadakis, E-mail: marios_papadakis@yahoo.gr Cite this article: Papadakis M. (2022). Why comparing coronavirus disease 2019 (COVID-19) and seasonal influenza fatality rates is like comparing apples to pears. Infection Control \& Hospital Epidemiology, 43: 958-959, https://doi.org/10.1017/ice.2021.140 of nearly $2.2 \%$. At the beginning, the fact that COVID-19 typically presents as a flu-like illness, led many healthcare professionals and scientists to adopt strategies traditionally used to fight seasonal influenza because both entities seemed to have similar patterns of viral shedding. This resemblance quickly resulted in direct comparisons of these separate entities in terms of fatality rates as well.

Although COVID-19 and seasonal influenza share several common clinical and epidemiological characteristics, a one-to-one comparison of fatality rates is not reliable. In fact, such 
a comparison can be dangerous in both directions. If a comparison of fatality rates is applied to countries with a strong containment of the COVID-19 pandemic, the severity of COVID-19 may be underrated, which can be potentially disastrous, especially in the presence of severe comorbidities. On the other hand, presenting severe acute respiratory coronavirus virus 2 (SARS-CoV-2) as a virus with much higher fatality rates can underrate the severity of the seasonal flu, with negative effects, for example, on flu vaccination rates.

According to Faust et al, ${ }^{1}$ the root of the confusion is a knowledge gap regarding how influenza and COVID-19 data are reported. Covid-19 data are actual numbers, whereas influenza data are only calculated estimates. This important point cannot be overstated. Faust et al compared COVID-19 death counts to influenza death counts over past seasons. They reported that a 1-week COVID-19 death rate in April 2020 was 9.5- to 44.1-fold greater than the peak week of influenza deaths during any of the past 7 influenza seasons. They also analyzed the case of Diamond Princess and reported that even the adjusted CFR of $0.5 \%$ "would still be 5 times the commonly cited CFR of adult seasonal influenza." Moreover, Faust et al concluded that to understand the true threat to public health from COVID-19, comparisons with seasonal influenza should be made using an apples-to-apples comparison. ${ }^{1}$

Although their facts are accurate, such comparisons may underrate the severity of seasonal influenza; rather, they are apples-topears comparisons. First and most importantly, COVID-19 is an ongoing pandemic, whereas influenza is largerly caused by endemic strains of several influenza virus subtypes that have circulated over decades as seasonal flu. These viral strains cause more or less severe epidemics annually. This evolving COVID-19 pandemic, caused by a new, previously unknown virus that has overwhelmed healthcare systems and caused shortage of medical supplies in almost every country of the world, cannot be directly compared to the seasonal outbreaks of an endemic disease caused by a well-studied virus for which vaccine protection is available for the main strains.

It would be more reasonable to compare 2 pandemics with each other. Several studies of this type exist; most compare the current pandemic with the 1918-19 influenza (Spanish flu) pandemic. He et al ${ }^{2}$ adopted the conventionally accepted CFR of $2 \%$ for 1918-19 influenza and reported comparable fatality rates in the United Kingdom. ${ }^{2}$ However, whether 2 pandemics that occurred 100 years apart can be compared on an apples-to-apples basis is also questionable. For example, suspected cases of the Spanish flu were not confirmed by laboratory tests and therefore the infection fatality rate (IFR; ie, proportion of deaths among all infected individuals of the Spanish flu) has been compared with the CFR (ie, proportion of death among individuals with laboratory-confirmed disease). Furthermore, fatality rates do not reflect the proportion of the world population infected. The Spanish flu infected $\sim 33 \%$ of the world population at the time.

Another candidate for a direct comparison could be the last global influenza pandemic, which occurred in 2009, due to the swine-origin influenza A virus subtype H1N1. According to the World Health Organization, the total number of laboratoryconfirmed pandemic A(H1N1) cases was $491,382,{ }^{3}$ including 18,449 deaths. ${ }^{4}$ This represents an overall CFR of $3.75 \%$, with a mean age at death of 37.4 years. ${ }^{5}$ The WHO emphasized "that the reported number of fatal cases is an under representation of the actual numbers as many deaths are never tested or recognized as influenzarelated." Although the CFR of the "fairly mild 2009 influenza pandemic" 5 is apparently much higher than that of the current COVID19 pandemic, such a comparison is, in fact, also debatable. For example, the WHO declared the counting of individual cases as no longer essential only a few months after declaring the $\mathrm{H} 1 \mathrm{~N} 1$ pandemic.

In summary, there is no need to directly compare COVID-19 and influenza in terms of fatality rates to prove the severity of the current pandemic. Both are harmful, dangerous, and potentially disastrous diseases, and they should be treated with the utmost respect.

Acknowledgments.

Financial support. No financial support was provided relevant to this article.

Conflicts of interest. All authors report no conflicts of interest relevant to this article.

\section{References}

1. Faust JS, Del Rio C. Assessment of deaths from covid-19 and from seasonal influenza. JAMA Intern Med 2020;180:1045-1046.

2. He D, Zhao S, Li Y, Cao P, Gao D, Lou Y, Yang L. Comparing COVID-19 and the 1918-19 influenza pandemics in the United Kingdom. Int J Infect Dis 2020;98:67-70.

3. Weekly virological update on 05 August 2010. World Health Organization website. https://www.who.int/csr/disease/swineflu/laboratory06_08_2010/ en/. Published 2010. Accessed December 23, 2020.

4. Pandemic (H1N1) 2009 - update 112. World Health Organization website. https://www.who.int/csr/don/2010_08_06/en/. Published 2010. Accessed Dec 23, 2020.

5. Petersen E, Koopmans M, Go U, et al. Comparing SARS-CoV-2 with SARS-CoV and influenza pandemics. Lancet Infect Dis 2020;20:e238-e244.

\title{
Uptake of treatment practice standards during a pandemic in an academic medical system
}

\author{
Maria Tate $\mathrm{MS}^{1}$, Jeannie D. Chan PharmD, MPH ${ }^{1,2}$, Christine Johnston MD, MPH${ }^{2}$, Shireesha Dhanireddy $\mathrm{MD}^{2}$, Margaret \\ L. Green MD, MPH ${ }^{2}$ and Rupali Jain PharmD ${ }^{1,2}$ (D) \\ ${ }^{1}$ University of Washington School of Pharmacy, Seattle Washington and ${ }^{2}$ Division of Allergy and Infectious Disease, University of Washington School of Medicine, \\ Seattle, Washington
}

\author{
Author for correspondence: Rupali Jain, E-mail: rupali@uw.edu \\ Cite this article: Tate M, et al. (2022). Uptake of treatment practice standards during a \\ pandemic in an academic medical system. Infection Control \& Hospital Epidemiology, 43: \\ 959-961, https://doi.org/10.1017/ice.2021.137
}

To the Editor - The first case of community spread of severe acute respiratory coronavirus virus 2 (SARS-CoV-2) in the United States was reported in Washington state in late January $2020 .{ }^{1}$ During the early stage of the pandemic, therapeutics were emerging at a rapid 